\title{
Development of a Low-Cost Extruded Scintillator with Co-Extruded Reflector for the MINOS Experiment
}

\author{
$\underline{\text { D.F. Anderson }}^{1}$, I. Ambats ${ }^{2}$, B. Baller ${ }^{1}$, P.M. Border ${ }^{3}$, B.C. Choudhary ${ }^{4}$, J.J. Grudzinski ${ }^{2}$, R. Heinz ${ }^{5}$, \\ N. Hill ${ }^{2}$, Y. Huang ${ }^{4}$, M. Ignatenko ${ }^{1}$, T. Joffe-Minor ${ }^{2}$, H.Y. Kim ${ }^{4}$, K. Lang ${ }^{6}$, V. Makeev ${ }^{1}$, K. Mellott ${ }^{1}$, \\ D.G. Michael ${ }^{4}$,L. Miller ${ }^{7}$, W.P. Oliver ${ }^{8}$, A. Para ${ }^{1}$, A. Pla-Dalmau ${ }^{1}$, K. Ruddick ${ }^{3}$, R.L. Talaga ${ }^{2}$, J. Trevor ${ }^{4}$, \\ J.C.Yun ${ }^{1}$
}

${ }^{1}$ Fermi National Accelerator Laboratory, PO Box 500, Batavia IL 60510

${ }^{2}$ Argonne National Laboratory, 9700 S. Cass Ave., Argonne IL 60439

${ }^{3}$ University of Minnesota, School of Physics and Astronomy, Minneapolis MN 55455

${ }^{4}$ California Institute of Technology, Pasadena CA 91125

${ }^{5}$ Indiana University, Bloomington IN 47405

${ }^{6}$ The University of Texas at Austin, Austin TX 78712

${ }^{7}$ James Madison University, Harrisonburg VA 22807

${ }^{8}$ Tufts University, Physics Dept., Medford MA 02155

\begin{abstract}
The MINOS experiment is a long-baseline, neutrinooscillation experiment. In total, $28,000 \mathrm{~m}^{2}$ of scintillator is needed for the experiment. This is almost 300 tons of finished scintillator. The solution has been the development of an extruded scintillator with a 2-mm deep grove in the upper surface for a wavelength-shifting fiber and a co-extruded $\mathrm{TiO}_{2}$ coating as a reflector. The $\mathrm{TiO}_{2}$ coating also allows the scintillator to be directly epoxied into panels. Production and quality control techniques are presented.
\end{abstract}

\section{INTRODUCTION}

MINOS is a long-baseline, neutrino-oscillation search experiment. The near detector, on the Fermilab site, measures the content and energy spectrum of the un-oscillated neutrino beam. The far detector, $735 \mathrm{~km}$ away in the Soudan Mine in northern Minnesota, also measures the energy spectrum of the beam. The energy spectra measured at the near and far detectors will be different if the neutrinos oscillate. Oscillation will indicate that neutrinos have mass.

The near and far detectors are large, tracking iron and scintillator calorimeters. Table I gives some of the experimental parameters of these detectors.

The experiment requires a large amount of scintillator at an affordable price. A total of $28,000 \mathrm{~m}^{2}$ of scintillator is needed, which is almost 300 tons of finished scintillator.
Table I

Some detector parameters of the MINOS experiment

\section{Parameter}

Near detector mass

Far detector mass

Steel plates (far detector)

Magnetic field (far detector)

Active scintillator strips

\author{
Value \\ 0.98 (metric) kt \\ 5.4 (metric) kt \\ 8-m wide, 2.54-cm thick octagons \\ Toroidal $1.5 \mathrm{~T}$ at $2 \mathrm{~m}$ radius \\ $4.1 \mathrm{~cm}$ wide, $1-\mathrm{cm}$ thick, $\sim 8-\mathrm{m}$
}

There were two scintillator options considered for the MINOS experiment: liquid scintillator and extruded scintillator. Both have their advantages and disadvantages. The decision was made to build on the experience in extruded, polystyrene scintillator at Fermilab [1-3]. The MINOS experiment thus developed an extruded scintillator with a coextruded reflective cap. The light from the scintillator is read out by a wavelength shifting (WLS) fiber that then is optically coupled to a clear fiber.

Each iron plane of the far detector is covered with eight scintillator modules. There are four modules that are 20 scintillator strips wide and four modules that are 28 scintillator strips wide (192 strips/plane). Over 105,000 strips must be read out, most on both ends. The readout is done by a wavelength-shifting fiber epoxied into a grove in the scintillator strips. The strips are epoxied in an aluminum box for strength and light tightness. The ends of the fibers are coupled to clear fibers that go to Hamamatsu multi-pixel 
photomultiplier tubes (PMT). Both ends of the fibers are read out with 8-fold multiplexing (eight fibers per PMT pixel). There are 1452 16-pixel PMT's and 210 64-pixel PMT in the experiment for a total of about 36,000 pixels

\section{II: MINOS SCINTILLATOR}

The profile of the MINOS scintillator strip is shown in figure 1. The cross sectional profile is $41 \mathrm{~mm}$ wide and 10 $\mathrm{mm}$ thick. There is a $2 \mathrm{~mm}$ deep groove in the top surface to accept the $1.2 \mathrm{~mm}$ (WLS) fiber. With the exception of the groove, the scintillator is covered with a $0.25 \mathrm{~mm}$ thick, coextruded reflective cap of $\mathrm{TiO}_{2}$ loaded polystyrene.

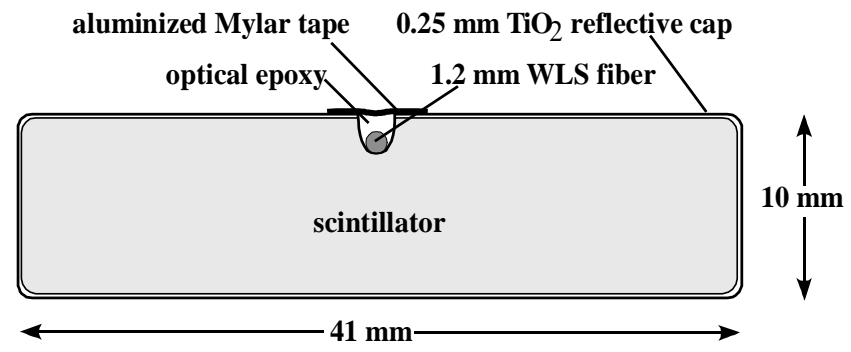

Figure 1: Profile of scintillator strip.

Because the light-travel distances between emission and absorption by the WLS fiber are relatively short, it was found that the light collection efficiency by the fiber was dominated by the reflectivity of the cap. Typical attenuation lengths of the scintillation light along the finished scintillator are $2-3 \mathrm{~cm}$. When the scintillator is made oxygen free, the attenuation length in the scintillator is secondary to the attenuation lengths of the WLS and clear fibers. This has allowed the use of relatively inexpensive, commercial grade (Dow 663) polystyrene pellets rather than expensive, optical-grade material (Dow 262).

There were significant savings made in the choice of dopants for the polystyrene. The primary dopant $(1 \%$ by weight) is PPO (2,5-diphenyloxazole), which cost about $\$ 125 / \mathrm{kg}$ as compared to PT (para-Terphenyl), which cost $\$ 200 / \mathrm{kg}$ or more. The secondary dopant $(0.03 \%$ by weight $)$ was chosen to be POPOP (1,4-bis(5-phenyloxazol-2yl)benzene) at $\$ 650 / \mathrm{kg}$ rather than DPS (transdiphenylstilbene) which cost $\$ 25,000-\$ 50,000 / \mathrm{kg}$. The final scintillator cost approximately $\$ 9 / \mathrm{kg}$ as compared to about $\$ 40 / \mathrm{kg}$ for cast material available on the market.

The extruded material also has advantages in labor savings over cast material. The scintillator needs only to have the WLS fiber epoxied into the groove and covered with reflective tape and it is ready to be epoxied into panels. The tough outer $\mathrm{TiO}_{2}$ coating on the extruded scintillator makes handling and fabrication of modules very easy.

\section{III: PERFORMANCE}

Figure 2 shows the average number of photoelectrons measured as a function of position for an early-prototype, 8meter long module, 20 scintillator strips wide. WLS fibers are routed from the end of the scintillator strips, via "manifolds", to an optical coupling.

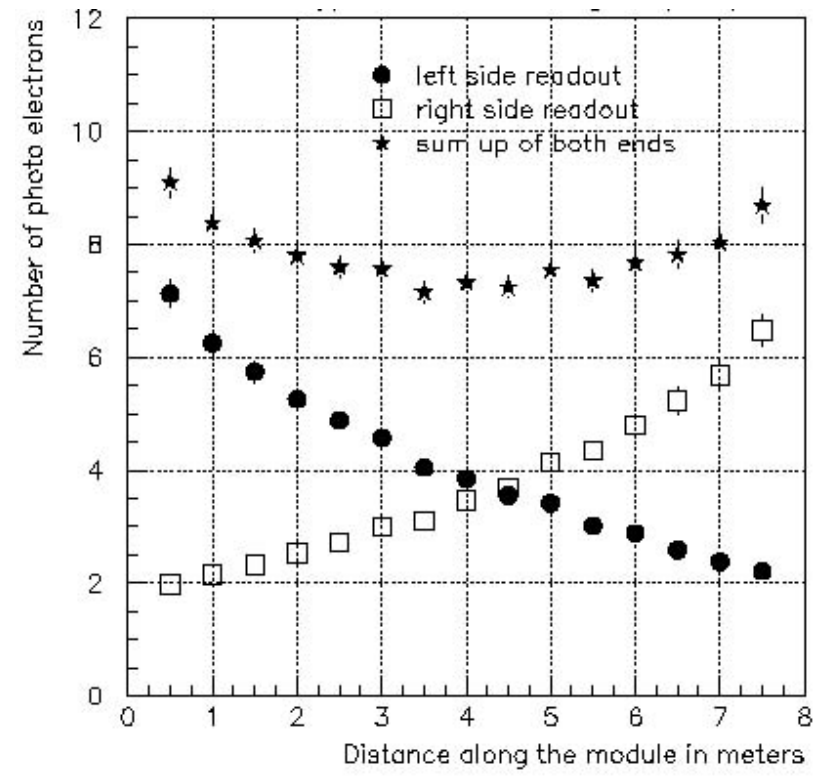

Figure 2: Averaged number of photoelectrons collected as a function of position.

The figure also shows the average number of photoelectrons measured from each end. For this module, the average number of photoelectrons (both ends summed) for a cosmic ray in the middle is about 7. A minimum ionizing particle through the near end of the module yields about 7 photoelectrons while yielding about 2 photoelectrons when measured from the far end. The WLS fibers vary in length but on the average they extend from each end of the scintillator by about one meter. There are also clear fibers, averaging $2 \mathrm{~m}$ in length connecting each end of the WLS fiber to the PMT. The attenuation lengths of the $1.2 \mathrm{~mm}$ diameter WLS and clear fibers used for readout are 5-6 $\mathrm{m}$ and 11-12 m, respectively.

Figure 3 shows the number of photoelectrons measured for each scintillator strip in the same module as in Figure 2. It should be noted that two of the strips were cut to a narrower width to correct for an error in width at the factory. These two strips are the strips with the least light output in Figure 3. Here one can easily see the variation from strip to strip. The strip-to-strip differences are caused by variation in the scintillator performance, irregularities in the position of the fiber and bubbles in the epoxy, occasional damage to the WLS fiber in the assembly process, and variations in the optical coupling in the connectors. Each of these variables contributes approximately $\pm 10 \%$ to the measured light output 
and is being addressed in future productions. The additional variable is the quantum efficiency in the PMTs. The response of the of the PMTs and the other variables mentioned above will be calibrated out in the final detectors.

\section{IV: EXTRUSION OF THE SCINTILLATOR}

\section{A. Preparation of material}

The key to producing good scintillator is to remove the water and oxygen from the materials. In particular it was found that oxygen causes oxidation of the scintillator in the

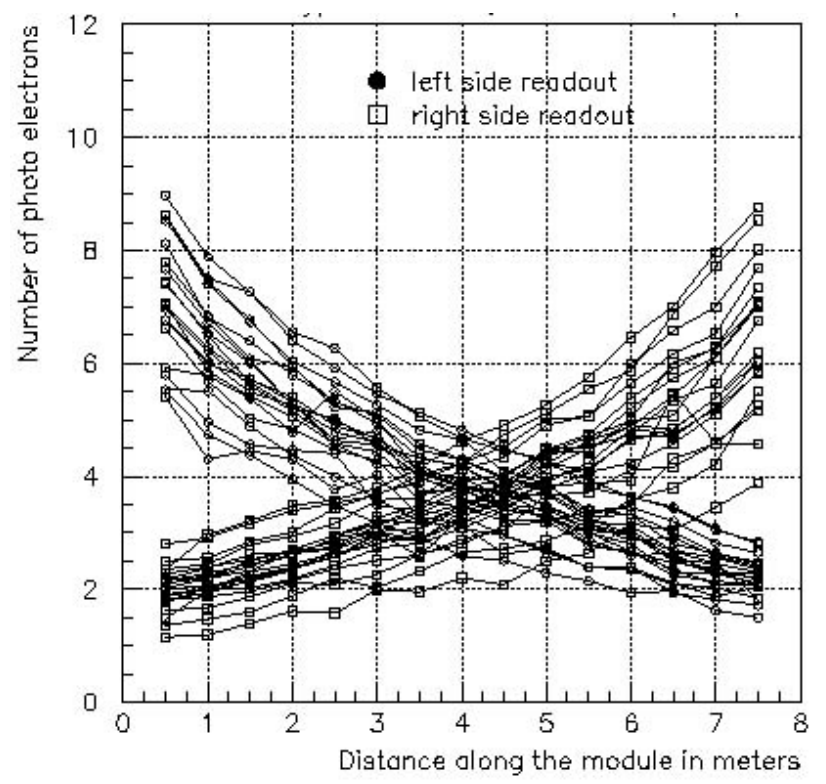

Figure 3: Averaged number of photoelectrons collected as a function of position for individual strips.

extruder turning it brown and greatly reducing the light output. The polystyrene pellets are dried for at least 4 hours at $77^{\circ} \mathrm{C}$, then partially cooled under a $\mathrm{N}_{2}$ purge in plastic-lined, steel drums for at least 4 hours. Under an $\mathrm{N}_{2}$ purge the premeasured dopants are added to the warm polystyrene pellets, in $45 \mathrm{~kg}$ batches, and mixed by tumbling for at least 15 minutes. The mixture is again returned to plastic-lined steel drums and purged with $\mathrm{N}_{2}$ for an additional 4 hours.

The mixture is then placed in the feed hopper for the extruder and kept under a $\mathrm{N}_{2}$ purge. The co-extruder is filled with a mixture of polystyrene pellets and polystyrene pellets that are loaded with $\mathrm{TiO}_{2}$. The mixture yields a reflective coating that is $12 \% \mathrm{TiO}_{2}$ by weight. The hopper of the coextruder is also kept under a $\mathrm{N}_{2}$ purge.

\section{B. Extrusion}

The main extruder is a batch-fed, 63-mm diameter, singlescrew extruder. This machine feeds, melts, mixes and delivers the scintillator core material to a profile die. A 32-mm diameter single-screw co-extruder is attached at 90 degrees to the profile die. This co-extruder injects the melted $\mathrm{TiO}_{2}$ and polystyrene mixture as reflective cap. The scintillator exits the die face close to the correct shape at approximately $200^{\circ} \mathrm{C}$. The material immediately enters vacuum-sizing tooling mounted in a $2.4-\mathrm{m}$ long chilled water/vacuum-sizing tank. Here, differential pressure draws the semi-molten material to the final dimensions. The material is then further cooled in an additional $2.4 \mathrm{~m}$ chilled water section. Upon exiting the cooling tank, the scintillator enters a belt puller, which maintains the line speed at about $2 \mathrm{~m} / \mathrm{min}$. For the MINOS profile, this line speed corresponds to material throughput of about $50 \mathrm{~kg} / \mathrm{hr}$. Exiting the puller, the material is fed into a traveling saw programmed to cut the scintillator to the desired length. The finished material is then subject to quality control checks and moved to storage.

\section{Quality Control}

In the development of a quality control (QC) technique the requirements were that it give real-time results, be able to be performed by the extruder operators, and could not require the use of a radioactive source at the factory. Many techniques were explored. They included measuring such parameters as the transmission of the bare extruded scintillator, the scintillation of the bare scintillator with a radioactive source (at Fermilab), the emission spectrum of the final product, and the reflectivity of the cap.

The solution was to measure at the factory the combined effect of the transmission through the finished scintillator and the reflectivity of the cap. The technique is illustrated in figure 4. Light peaking at $450 \mathrm{~nm}$ is introduced through the groove by optical fibers at about $8 \mathrm{~cm}$ from one end of the 14 $\mathrm{cm}$ long sample. The intensity of the transmitted light is then measured from the end. The end was prepared by simply removing the saw cuts with a file. A modified fluorescence spectrometer (Hitachi model F2500) was used for the purpose.

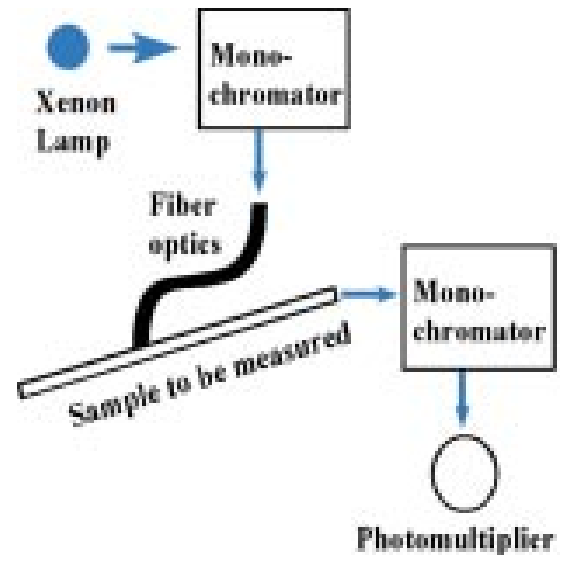

Figure 4: Schematic of QC layout 
The response of a reference sample is first measured and followed by the response of the sample to be measured. The scintillation light output of the reference sample is verified with either a radioactive source or with cosmic rays beforehand. The ratio of intensities of the reference sample and the sample to be measured is approximately equal to the ratio of intensities of the transmitted light from the two pieces. An exception is discussed below. The reference sample used at the factory was chosen to have the minimum acceptable response, so that anything with a greater transmission would have greater light output. One caveat discussed below is that this assumes that there is no problem with the dopant concentration. Figure 5 shows a comparison of the estimated light output from the UV transmission measurements made at the factory and measurements made at Fermilab on the same samples with a radioactive source. The vertical scale of the UV measurement is shifted by $10 \%$ from that of the source measurement to correspond to a probable error in calibration at the factory. The light output of several of the samples were also measured with cosmic rays as a cross check. The agreement is quite good considering the environment the UV transmission measurements were made in and the skill level of the people making the measurements.

The UV transmission measurements are very useful for the pass/fail determination needed at the factory. The measurements also allowed the operator of the extruder to quickly spot troubles, such as running out of nitrogen on one of the flows. One shortcoming of this particular QC check is that it is not sensitive to problems with the dopants. In fact, material made without any dopants would have a very high transmission. So an additional test, sensitive to possible problems with the dopants, was developed.

This second test for dopants, performed at Fermilab, entails fluorescing the ends of the samples with $320 \mathrm{~nm} \mathrm{UV}$ light and examining the emission spectra. Figure 6 shows the fluorescence spectra from three samples with measured light outputs of $164 \%, 84 \%$, and $50 \%$ of a reference sample. All three spectra were normalized in amplitude to peak "A", which is a PPO peak. The ratio of a POPOP peak " $\mathrm{B}$ " is then measured and if the ratio is below 1.2 the sample and those around it are evaluated with a radioactive source. In three weeks of a recent production run, three $45-\mathrm{kg}$ lengths of extrusion were identified as being bad. This is about $1 \%$ of the three-week's production. It was believed that the problem was in the mixing of the dopants. This test takes about 3.5 hours to evaluate the samples from a 1-week, $24 \mathrm{hr} /$ day production and is a good backup to the UV measurement performed at the factory.

\section{Final Product}

At the time of this writing, the MINOS experiment has had a 3 -week production run of scintillator. That is a

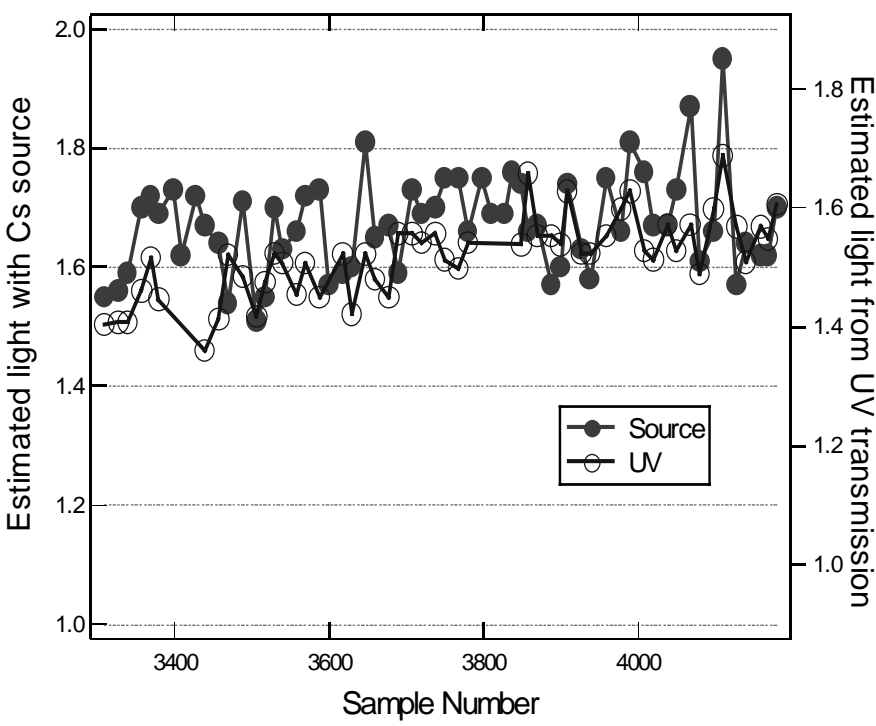

Figure 5: Comparison of QC measuremts made at extrusion factory with UV source and at Fermilab with a radioactive source.

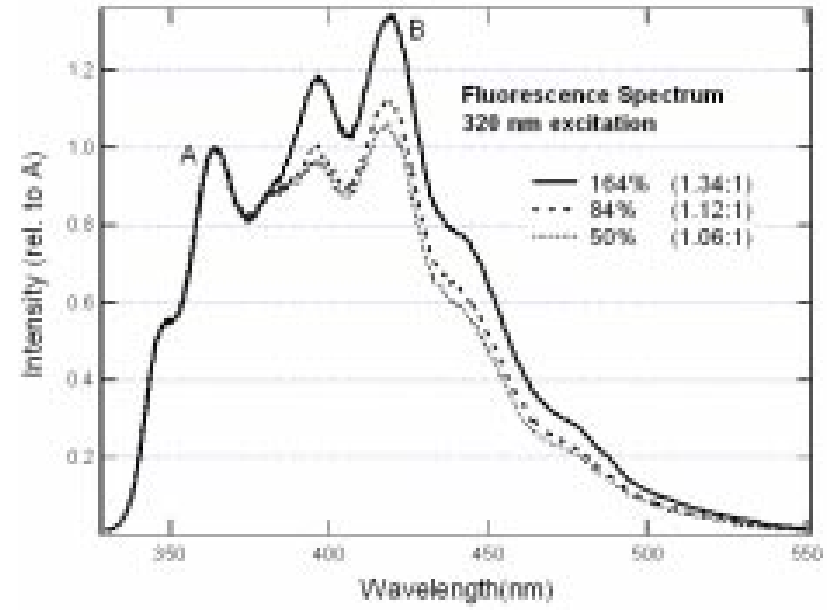

Figure 6: Emission spectra for three samples of scintillator.

production of about 15 ton (about $40 \mathrm{~km}$ ) of scintillator. With the exception of the three- $45 \mathrm{~kg}$ lengths of extrusions that displayed a dopant problem, the entire production had very good light output. The typical light output of the scintillator produced is as much as $50 \%$ higher than the material used in the module of Figure 2.

One point of concern before the production was the uniformity of the dimensions of the extrusion. Figure 7 shows the average width of the extrusion, along with the standard deviation, by day, for the three-weeks of production. The specification for the width given to the manufacturer was $41 \pm$ $0.5 \mathrm{~mm}$. Clearly they were able to do much better than that. There was a similar small spread in the dimensions of the height of the scintillator. 


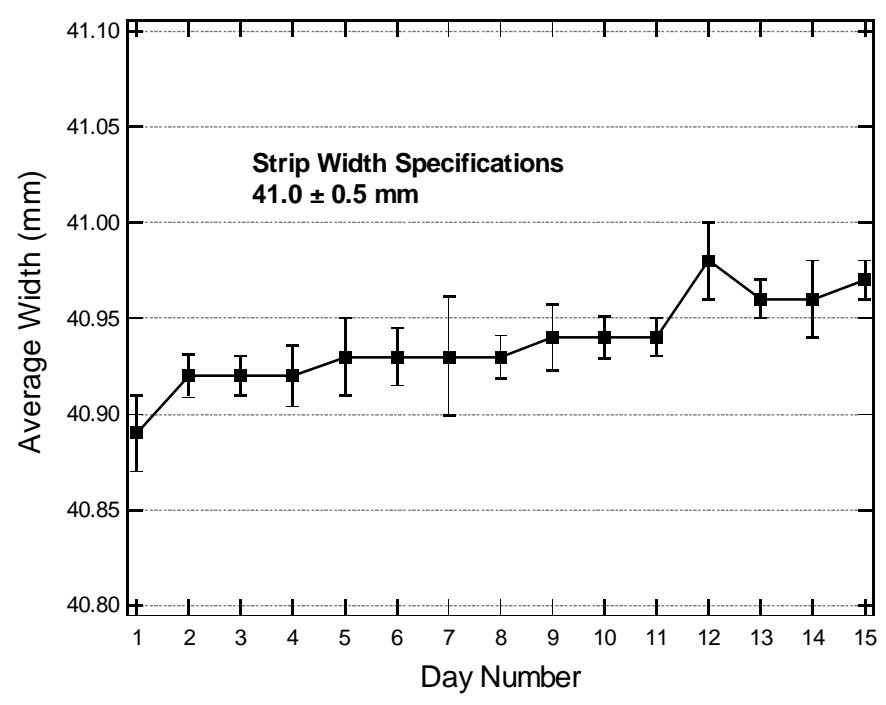

Figure 7: Average scintillator width by day.

There was a minor problem with variations in the dimensions of the groove. This was due to a problem with the die. This problem has already been corrected.

\section{CONCLUSIONS}

It has been demonstrated that extruded scintillator can be produced with a co-extruded reflective coating in an industrial environment. The material is much less expensive than cast scintillator, and much easier to work with. The quality of the extruded scintillator has proven to be high and easily read out with WLS fibers. There were also good mechanical tolerances maintained.

Techniques for QC have been developed which allows the factory to monitor the quality of the scintillator in almost real time. This is done with simple modifications to commercially available instruments, and with little training requirements at the factory. There are also no radioactive sources used at the factory. This is a technique suitable for future large calorimeter.

\section{REFERENCES:}

[1] A. Pla-Dalmau, A.D. Bross and K. Mellott, "Extruded Plastic Scintillation Detectors", January 1999 DPF Proceedings (Fermilab-Conf-99/095)

[2] A. Pla-Dalmau, A.D. Bross and K. Mellott, "Low-Cost Extruded Plastic Scintillator", (Fermilab-Pub-00/177-E) Submitted to NIMA.

[3] The MINOS detectors, Technical Design Report, NuMIL-337 (1998) 